une revue Gallia

Rhône-Alpes | 2008

\title{
Seez
}

Col du Petit Saint-Bernard

\section{Sylvie Crogiez-Pétrequin}

\section{(2) OpenEdition}

12 Journals

Édition électronique

URL : http://journals.openedition.org/adlfi/1570

ISSN : 2114-0502

Éditeur

Ministère de la culture

Référence électronique

Sylvie Crogiez-Pétrequin, «Seez », ADLFI. Archéologie de la France - Informations [En ligne], Rhône-Alpes, mis en ligne le 01 mars 2008, consulté le 19 avril 2019. URL : http://journals.openedition.org/ adlfi/1570

Ce document a été généré automatiquement le 19 avril 2019

(c) Ministère de la Culture et de la Communication, CNRS 


\title{
Seez
}

\author{
Col du Petit Saint-Bernard
}

\section{Sylvie Crogiez-Pétrequin}

\section{Identifiant de l'opération archéologique : 9791}

Date de l'opération : 2008 (FP)

1 La dernière campagne de fouilles sur le bâtiment ouest s'est déroulée de la mi-juillet à la mi-aout, sans interruption. Son objectif a été pleinement rempli : déterminer si nous étions en présence de fondations ou d'élévation et rendre lisible le bâtiment aussi bien en plan qu'en volume, tout en avançant dans la datation du fonctionnement du site.

Une première zone de fouilles a été implantée dans un espace déjà en partie exploré en 2004 (secteur 2008-2) où l'on avait découvert une brique posée horizontalement et une couche rubéfiée, voire brûlée, le long du mur 222 (voir le plan du bâtiment avec les numéros des murs : fig. 38, p. 212, BSR 2005). La brique a subi son dernier chauffage entre

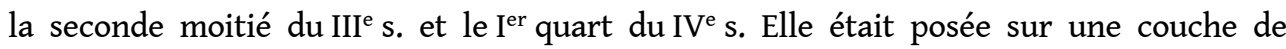
démolition incendiée très épaisse, dont la dernière strate contenait du mobilier en quantité importante ( $\mathrm{du} \mathrm{III}^{\mathrm{e}} \mathrm{s}$. surtout) et du charbon de bois qui a été daté entre 132 et 263. Le secteur a révélé aussi la présence d'un tronçon de mur ou d'une dalle de calcaire qui a éclaté sous la chaleur de l'incendie dans ce secteur. On peut noter que c'est seulement dans cette zone de l'édifice, qui n'avait jamais été fouillée, qu'on a pu relever des traces d'incendie comme en 2006. Le bâtiment était encore utilisé au moins en partie jusqu'à la fin du III ${ }^{\mathrm{e}} \mathrm{s}$.

3 Le secteur 2008-5 a été ouvert à l'est de la barrière antichars afin de finir de déterminer les limites de l'édifice et de confirmer les observations faites en 2006 sur la technique de construction et de protection des murs contre le climat du col. Comme en 2006, on a observé la présence d'une couche de dalles de schiste posées de chant contre le mur sur l'extérieur de l'édifice. Il est probable cependant que ces dalles et le mur mis au jour correspondent aux fondations plutôt qu'aux élévations. 
le secteur 2008-4, à l'extrémité sud de la cour centrale, proche des canalisations mises au jour en 2004, l'objectif essentiel était de comprendre l'agencement des murs 215 et 216, dans une zone perturbée par une tranchée de 1991. Il a livré un mobilier en quantité importante parmi lequel des monnaies et une tuile estampillée. Il est aujourd'hui vraisemblable de restituer un mur fermant la cour vers le sud, de la même façon qu'on pourrait le restituer au nord. Les vestiges des murs sont conservés sur une hauteur relativement importante, avec une différence toutefois entre le côté interne (hauteur plus importante) et externe du mur.

5 Un dernier secteur de fouille a été ouvert à l'intérieur du bâtiment, au sud (secteur 2008-3). La couche d'abandon présente sur l'ensemble du secteur a été perturbée par des aménagements contemporains, (vraisemblablement militaires et datant de la seconde guerre mondiale. Elle date des alentours de 230. Les murs 214, 215 et 218 sont très bien conservés sur une hauteur de plus de 2,50 m à l'intérieur de l'édifice, mais il s'agit ici de fondations même si elles sont parementées; trois sondages l'ont mis en évidence sans discussion. Le mur 212 est en fait la restauration du mur 214 ; il est beaucoup moins large que le mur d'origine (214). Les murs 215 et 218 ont été construits en même temps et on les a contrefortés grâce à un apport de dalles de schiste sur une petite largeur (1 m maximum) tant à l'extérieur qu'à l'intérieur de l'édifice. Ces dalles de schiste marquent le passage des fondations à l'élévation parementée. Cette technique de construction originale est sans doute régionale, et plus exactement montagnarde. On n'a pour l'instant trouvé aucun autre exemple d'époque romaine illustrant cette technique.

Le mobilier (céramique, instrumentum,monnaies, faune de 2006 et 2008) a été complètement inventorié et étudié. On note les mêmes ensembles datants que pour les campagnes précédentes (début $\mathrm{I}^{\mathrm{er}} \mathrm{s}$. après et III $\mathrm{s}$.). La campagne 2008 a cependant livré un nombre impressionnant de monnaies (45), du III $\mathrm{e}$ s. pour leur grande majorité. En ce qui concerne la céramique, on note toujours aussi peu d'amphores ( $5 \%$ des NMI). Cette campagne a été aussi marquée par la mise au jour de tuiles estampillées RPA et PUBLI/ PUBLIO ; dont quelques exemplaires semblent avoir été trouvés par P. Barocelli au début $\mathrm{du} \mathrm{XX}^{\mathrm{e}} \mathrm{s}$. Une étude plus précise est en cours. Un plan général de l'édifice est en cours d'élaboration (en plan et en volume). Enfin le mortier des contreforts (US 236) mis au jour en 2006 a été daté en laboratoire et serait probablement d'époque contemporaine, comme on le suspectait au moment de la fouille. Ces contreforts ont sans doute été construits par les fouilleurs qui ont mis au jour le mur nord de l'édifice afin de le préserver.

7 Le bâtiment ouest a donc été construit au début du premier siècle de notre ère, vraisemblablement une première fois abandonné et en ruine dans le courant du $\mathrm{III}^{\mathrm{e}} \mathrm{s}$., et sporadiquement encore utilisé par la suite puisqu'on y retrouve du mobilier du IV ${ }^{e} \mathrm{~s}$. Sa fonction précise au delà de l'accueil des voyageurs, reste toujours une énigme, et ne pourra sans doute être déterminée que si les fouilles sur les vestiges situés sur le versant italien du col reprennent, dans la mesure où l'on pourrait alors comparer les résultats pour les deux édifices les plus importants d'Alpis graia. 
INDEX

operation Fouille programmée (FP)

Index chronologique : Ier siècle apr. J.-C., IIe siècle apr. J.-C., IIIe siècle apr. J.-C., IVe siècle apr. J.-C.

Index géographique : Rhône-Alpes, Savoie (73), Séez

Thèmes : amphore, céramique, col large, estampille, habitat, marque sur tuile, monnaie romaine, passage, tuile

\section{AUTEUR}

SYLVIE CROGIEZ-PÉTREQUIN

université 